\title{
FEATURE EVALUATION FOR BUILDING FACADE IMAGES — AN EMPIRICAL STUDY
}

\author{
Michael Ying Yang ${ }^{a}$, Wolfgang Förstner ${ }^{b}$, Dengfeng Chai ${ }^{c}$ \\ ${ }^{a}$ Institute for Information Processing (TNT), Leibniz University Hannover \\ ${ }^{b}$ Department of Photogrammetry, Institute of Geodesy and Geoinformation, University of Bonn \\ ${ }^{c}$ Institute of Spatial Information Technique, Department of Earth Science, Zhejiang University \\ yang@tnt.uni-hannover.de, wf@ipb.uni-bonn.de, chaidf@cad.zju.edu.cn
}

\section{Working Groups ICWG III/VII}

KEY WORDS: Feature, evaluation, random decision forest, facade image

\begin{abstract}
:
The classification of building facade images is a challenging problem that receives a great deal of attention in the photogrammetry community. Image classification is critically dependent on the features. In this paper, we perform an empirical feature evaluation task for building facade images. Feature sets we choose are basic features, color features, histogram features, Peucker features, texture features, and SIFT features. We present an approach for region-wise labeling using an efficient randomized decision forest classifier and local features. We conduct our experiments with building facade image classification on the eTRIMS dataset, where our focus is the object classes building, car, door, pavement, road, sky, vegetation, and window.
\end{abstract}

\section{INTRODUCTION}

Despite the substantial advances made during the past decade, the classification of building facade images remains a challenging problem that receives a great deal of attention in the photogrammetry community (Rottensteiner et al. 2007, Korč and Förstner. 2008; Fröhlich et al. 2010, Kluckner and Bischof, 2010, Teboul et al. 2010). Image classification is critically dependent on the features. Typical feature evaluation can be divided into two stages. First, image processing is used to extract a set of robust features that implicitly contains the information needed to make classspecific decisions while resisting extraneous effects such as changing object appearance, pose, illumination and background clutter. Second, a machine learning based classifier uses the features to make region-level decisions, often followed by post-processing to merge nearby decisions. Instead of using some unsupervised techniques, which bare generalization problem, it is popular way that the classifier is trained using a set of labeled training examples. The overall performance depends critically on all three elements: the feature set, the classifier \& learning method, and the training set. In this paper, we focus on evaluating different feature sets.

Korč and Förstner (2009) published an image dataset showing urban buildings in their environment. It allows benchmarking of facade image classification, and therefore the repeatable comparison of different approaches. Most of the images of this data set show facades in Switzerland and Germany. Regarding terrestrial facade images, the most dominant objects are the building itself, the window, vegetation, and the sky. Fig. 1 demonstrates the variability of the object data.

In this work, we empirically investigate extended feature sets on eTRIMS dataset (Korč and Förstner, 2009). We show random forest gives some reasonable classification results on building facade images, and evaluate classification results by counting corrected labeled regions. The remainder of the paper is organized as follow. Section 2 reviews some existing methods for feature evaluation and building facade image classification. Then, we introduce feature sets for evaluation in the scope of the paper in Section 3 Randomized decision forest classifier for performing image classification is described in Section 4 In Section 5 , we

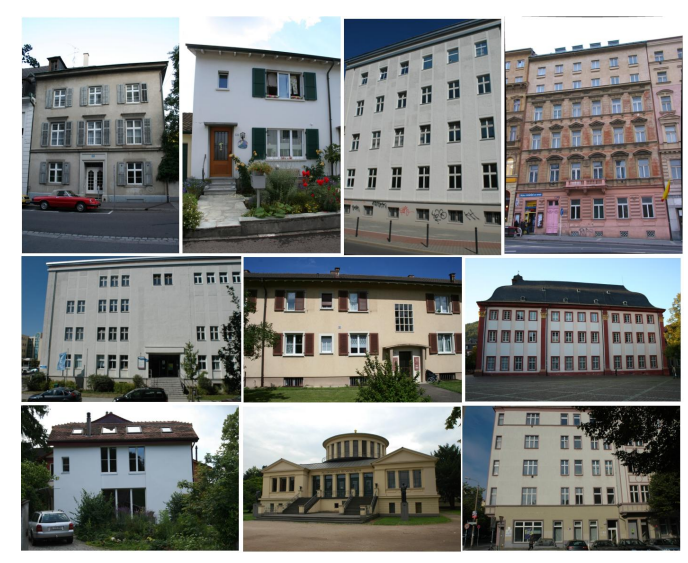

Figure 1: Example images from benchmark data set (Korč and Förstner 2009).

show our results and discuss the effect of each feature sets with respect to the classification of facade images. We finally conclude with a brief summary in Section 6

\section{RELATED WORKS}

Previous works on building facade classification mostly regard the facade classification problem as multiple object detection tasks. Building facade detection is a very active research area in photogrammtery and computer vision. A feature selection scheme with Adaboost for detecting buildings and building parts is presented in Drauschke and Förstner (2008). In recent approaches, graphical models are often used for integrating further information about the content of the whole scene (Kumar and Hebert. 2003, Verbeek and Triggs, 2007). In another paradigm, the bag of words, objects are detected by the evaluation of histograms of basic image features from a dictionary (Sivic et al. 2005). Unfortunately, both approaches have not been tested with high resolution building images. Furthermore, the bag of words approaches have not applied to multifarious categories as building, and it is extremely slow and often the most time consuming part of the 
whole system, even with optimizations such as kd-trees, or hierarchical clusters (Nister and Stewenius, 2006).

Support vector machine (SVM) is widely considered as a good classifier. Schnitzspan et al. (2008) propose hierarchical support vector random fields that SVM is used as a classifier for unary potentials in conditional random field framework. While the training and cross-validation steps in SVM are time consuming, randomized decision forest (RDF) (Breiman, 2001) is introduced to significantly speed up the learning and prediction process. Existing work has shown the power of a randomized decision forest as a classifier (Bosch et al., 2007, Lepetit et al., 2005. Maree et al. 2005). The use of a randomized decision forest for semantic segmentation was previously investigated in Shotton et al. (2008); Dumont et al. (2009); Fröhlich et al. (2010). These approaches utilize simple color histogram features or pixel differences. Fröhlich et al. (2010) present an approach using an randomized decision forest and local opponent-SIFT features (van de Sande et al. 2010) for pixelwise labeling of facade images. Teboul et al. (2010) perform multi-class facade segmentation by combining a machine learning approach with procedural modeling as a shape prior. Generic shape grammars are constrained so as to express buildings only. Randomized forests are used to determine a relationship between the semantic elements of the grammar and the observed image support. Drauschke and Mayer (2010) also use random forest as one of the classifiers to evaluate the potential of seven texture filter banks for the pixelbased classification of terrestrial facade images.

\section{FEATURE SETS}

Features contains the information needed to make the class-specific decisions while being highly invariant with respect to extraneous effects such as changing object appearance, pose, illumination and background clutter. Advances in feature sets have been a constant source of progress over the past decade. Several well-engineered features have been experimentally found to be well fit for image classification task (Drauschke and Mayer. 2010). In this work, we derive 6 feature sets from each region obtained from some unsupervised segmentation algorithms, such as mean shift (Comaniciu and Meer 2002), watershed (Vincent and Soille 1991), or graph-based method (Felzenszwalb and Huttenlocher 2004).

Basic features $\boldsymbol{f}_{1} \quad$ First feature set $\boldsymbol{f}_{1}$ are basic features including (1). number of components of the region $(C)$; (2). number of holes of the region $(H)$; (3). Euler characteristic for planar figures (Lakatos, 1976) $(E=C-H)$; (4). area $(A)$; (5). perimeter $(U)$; (6). form factor $\left(F=U^{2} /(4 \pi A)\right)$; (7). height of bounding box; (8). width of bounding box; (9). area ratio between region and its bounding box; (10). height portion of center; (11). width portion of center.

Color features $\boldsymbol{f}_{2} \quad$ For representing spectral information of the region, we use 9 color features (Barnard et al. 2003) as second feature set $\boldsymbol{f}_{2}$ : the mean and the standard deviation of R-channel, G-channel and B-channel respectively in the RGB color space; and the mean of $\mathrm{H}$-channel, S-channel and $\mathrm{V}$-channel respectively in the HSV color space.

Histogram features $f_{3}$ We also include features derived from the gradient histograms as third feature set $\boldsymbol{f}_{3}$, which has been proposed by Korč and Förstner (2008). We determine gradient and its orientation and its magnitude. The histograms are determined for the 3 colors $\mathrm{R}, \mathrm{G}$ and $\mathrm{B}$ respectively in the region. Then, we derive the mean, the variance and the entropy from each histogram as features.
Peucker features $\boldsymbol{f}_{4}$ Peucker features are derived from generalization of the region's border as fourth feature set $\boldsymbol{f}_{4}$, and represent parallelity or orthogonality of the border segments. We select the four points of the boundary which are farthest away from each other. From this polygon region with four corners, we derive 3 central moments, and eigenvalues in direction of major and minor axis, aspect ratio of eigenvalues, orientation of polygon region, coverage of polygon region, and 4 angles of polygon region boundary points.

Texture features $\boldsymbol{f}_{5}$ We use texture features derived from the Walsh transform (Petrou and Bosdogianni, 1999, Lazaridis and Petrou. 2006) as fifth feature set $\boldsymbol{f}_{5}$, as features from Walsh filters are among the best texture features from the filter banks (Drauschke and Mayer. 2010). We determine the magnitude of the response of 9 Walsh filters. For each of the 9 filters, we determine mean and standard deviation for each region.

SIFT features $\boldsymbol{f}_{6} \quad$ Sixth feature set $\boldsymbol{f}_{6}$ are mean SIFT (ScaleInvariant Feature Transform) descriptors (Lowe 2004) of the image region. SIFT descriptors are extracted for each pixel of the region at a fixed scale and orientation, which is practically the same as the HOG descriptor (Dalal and Triggs. 2005), using the fast SIFT framework in Vedaldi and Fulkerson (2008). The extracted descriptors are then averaged into one $l_{1}$-normalized descriptor vector for each region.

These features are roughly listed in Table 1 The resulting 178 features are then concatenated into one feature vector.

Table 1: List of derived features from image regions. The number indicates feature numbers in each feature set.

\begin{tabular}{|l|}
\hline $\boldsymbol{f}_{1}$ basic features (11) \\
region area and perimeter, compactness and aspect ratio, etc. \\
\hline $\boldsymbol{f}_{2}$ color features (9) \\
mean and standard deviation of RGB and HSV spaces \\
\hline $\boldsymbol{f}_{3}$ histogram features $(9)$ \\
mean, variance and entropy of histogram from the gradients \\
\hline $\boldsymbol{f}_{4}$ Peucker features (12) \\
moments and eigenvalues of a region \\
\hline $\boldsymbol{f}_{5}$ texture features (18) \\
texture features derived from the Walsh transform \\
\hline $\boldsymbol{f}_{6}$ SIFT features (128) \\
mean SIFT descriptor features \\
\hline
\end{tabular}

\section{RANDOMIZED DECISION FOREST}

Features are evaluated by a classifier which operates on the regions defined by unsupervised segmentation. we take randomized decision forest (RDF) (Breiman 2001) as the classifier for performing feature evaluation, where the derived features from the image regions for the RDF classifier are chosen from Table 1 Existing work has shown the power of decision forests as the classifiers (Maree et al. 2005: Lepetit et al., 2005: Bosch et al. 2007). As illustrated in Fig. 2, a RDF is an ensemble classifier that consists of $T$ decision trees (Shotton et al. 2008). The feature vector of image region is classified by going down each tree. This process gives a class distribution at the leaf nodes and also a path for each tree. The class distribution is obtained by averaging the class distribution over the leaf nodes for all $T$ trees. This classification procedure is identical to Shotton et al. (2008).

In order to train the RDF classifier, we take the ground-truth label of each region to be the majority vote of the ground-truth pixel 


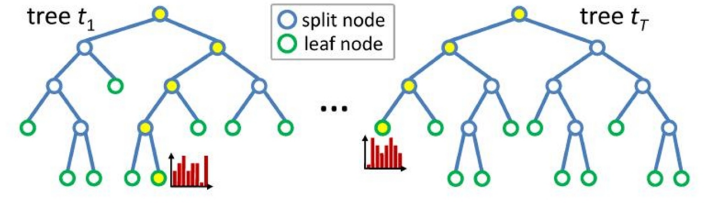

Figure 2: Decision forest. A forest consists of $T$ decision trees. A feature vector is classified by descending each tree. This gives, for each tree, a path from root to leaf, and a class distribution at the leaf. As an illustration, we highlight the root to leaf paths (yellow) and class distributions (red) for one input feature vector. (Figure courtesy by Jamie Shotton (Shotton et al. 2008).)

labels. Then a RDF is trained on the labeled data for each of the classes. According to a decision tree learning algorithm, a decision tree recursively splits left or right down the tree to a leaf node. We use the extremely randomized trees (Geurts et al. 2006) as learning algorithm. Each tree is trained separately on a small random subset of the training data. The learning procedure is identical to Shotton et al. (2008). We refer the reader to Shotton et al. (2008) for more details.

\section{EXPERIMENTAL RESULTS}

We conduct experiments to evaluate the performance of different image feature sets on the eTRIMS 8-class dataset (Korč and Förstner 2009). In the experiments, we take the ground-truth label of a region to be the majority vote of the ground-truth pixel labels. We randomly divide the images into training and test data sets.

\subsection{Experimental Setup}

We start with the eTRIMS 8-class dataset which is a comprehensive and complex dataset consisting of 60 building facade images, mainly taken from Basel, Berlin, Bonn, and Heidelberg, labeled with 8 classes: building, car, door, pavement, road, sky, vegetation, window. These classes are typical objects which can appear in images of building facades. The ground-truth labeling is approximate (with foreground labels often overlapping background objects). In the experiments, we randomly divide the images into a training set with 40 images and a testing set with 20 images.

Features are evaluated by the RDF classifier which operates on the regions defined by unsupervised segmentation. Therefore, the initial unsupervised segmentation algorithms may play an important role in the final classification results. To test how much the influence of the segmentation algorithms would be, we employ two unsupervised segmentation methods to segment the facade images, namely the mean shift algorithm (Comaniciu and Meer 2002) and the watershed algorithm (Vincent and Soille 1991).

First, we segment the facade images using mean shift algorithm (Comaniciu and Meer 2002), tuned to give approximately 480 regions per image. In all 60 images, we extract around 29600 regions. We have following statistics. Compared to the ground truth labelling, almost $36 \%$ of all the segmented regions get the class label building. $26 \%$ of all regions get the class label window. These statistics are very comprehensive, because facade images show buildings typically contain many windows. Furthermore, $21 \%$ of the regions get the class label vegetation, and $2 \%$ belong to $s k y$, and the last $15 \%$ of the regions are spread over most of other classes. Table 2 summarizes the statistics for the percentage of each class label, the average size of the region of each class, and the percentage of the image covered by each class for the baseline mean shift segmentation on the eTRIMS 8-class dataset (Korč and Förstner. 2009).

Table 2: Statistics of the percentage of each class label, the average size of the region of each class, and the percentage of the image covered by each class for the baseline mean shift segmentation on the eTRIMS 8-class dataset. $(\mathrm{b}=$ building, $\mathrm{c}=$ car, $\mathrm{d}=$ door, $\mathrm{p}=$ pavement, $\mathrm{r}=$ road, $\mathrm{s}=$ sky, $\mathrm{v}=$ vegetation, $\mathrm{w}=$ window, same for all the following tables and figures.)

\begin{tabular}{|l|rrrrrrrr|}
\hline & \multicolumn{1}{|c|}{ Mean shift } & & \\
& $\mathrm{b}$ & $\mathrm{c}$ & $\mathrm{d}$ & $\mathrm{p}$ & $\mathrm{r}$ & $\mathrm{s}$ & $\mathrm{v}$ & w \\
\hline \hline class percentage & 36 & 5 & 2 & 2 & 2 & 2 & 21 & 26 \\
\hline average size of region & 1014 & 424 & 569 & 1671 & 2563 & 6741 & 380 & 310 \\
\hline class covering percentage & 48 & 3 & 1 & 4 & 6 & 16 & 11 & 11 \\
\hline
\end{tabular}

Then, we segment the images using watershed algorithm (Vincent and Soille 1991, which turns out to give approximately 900 regions per image. In all 60 images, we extract around 56 000 regions. We have following statistics. Almost $34 \%$ of all the segmented regions get the class label building. $28 \%$ of all regions get the class label window. Furthermore, 23\% of the regions get the class label vegetation, $2 \%$ belong to sky, and the last $13 \%$ of the regions are spread over most of other classes. Table 3 summarizes the statistics for the percentage of each class label, the average size of the region of each class, and the percentage of the image covered by each class for the baseline watershed segmentation on the eTRIMS 8-class dataset (Korč and Förstner, 2009).

Table 3: Statistics of the percentage of each class label, the average size of the region of each class, and the percentage of the image covered by each class for the baseline watershed segmentation on the eTRIMS 8-class dataset.

\begin{tabular}{|l|rrrrrrrr|}
\hline & \multicolumn{1}{|c}{ Watershed } & & \\
& $\mathrm{b}$ & $\mathrm{c}$ & $\mathrm{d}$ & $\mathrm{p}$ & $\mathrm{r}$ & $\mathrm{s}$ & $\mathrm{v}$ & $\mathrm{w}$ \\
\hline \hline class percentage & 34 & 4 & 1 & 2 & 2 & 2 & 23 & 28 \\
\hline average size of region & 614 & 268 & 477 & 684 & 1490 & 4096 & 209 & 152 \\
\hline class covering percentage & 49 & 3 & 1 & 4 & 6 & 16 & 11 & 10 \\
\hline
\end{tabular}

\subsection{Evaluation with mean shift segmentation and RDF clas- sifier}

In the following, we first evaluate with RDF classifier on each feature set $\boldsymbol{f}_{1}, \boldsymbol{f}_{2}, \boldsymbol{f}_{3}, \boldsymbol{f}_{4}, \boldsymbol{f}_{5}$, and $\boldsymbol{f}_{6}$. Then, we evaluate with RDF classifier on the combination of feature sets, and show that RDF gives some reasonable results on building facade images.

The overall classification accuracy is listed in Table 4, when applying RDF classifier on each feature set. The number of decision trees is chosen as $T=250$. In all the following experiments, we always assume maximum depth of each decision tree $D=7$. A random classifier for 8 classes, the expected classification accuracy is $12.5 \%$. Fig. 3 shows the corresponding classification results over all 8 classes. Each class is normalized to $100 \%$.

Table 4: Average accuracy of RDF classifier on each feature set of eTRIMS 8-class dataset.

\begin{tabular}{l|llllll}
\hline feature set & $\boldsymbol{f}_{1}$ & $\boldsymbol{f}_{2}$ & $\boldsymbol{f}_{3}$ & $\boldsymbol{f}_{4}$ & $\boldsymbol{f}_{5}$ & $\boldsymbol{f}_{6}$ \\
\hline accuracy & $43.8 \%$ & $49.6 \%$ & $36.5 \%$ & $40.9 \%$ & $27.9 \%$ & $54.1 \%$ \\
\hline
\end{tabular}




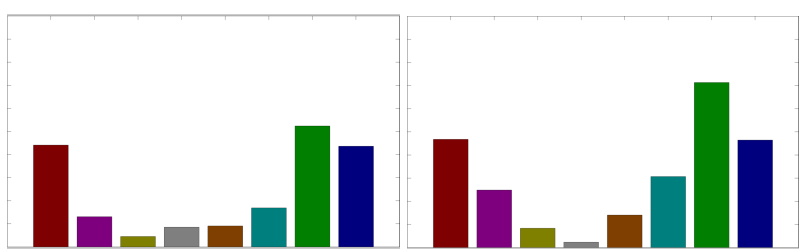

(a) On feature set $\boldsymbol{f}_{1}$ (Left), $\boldsymbol{f}_{2}$ (Right)

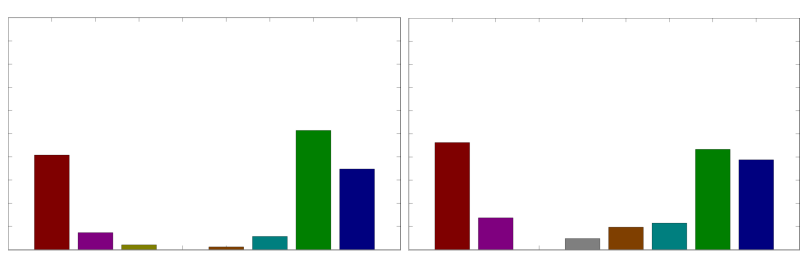

(b) On feature set $\boldsymbol{f}_{3}$ (Left), $\boldsymbol{f}_{4}$ (Right)

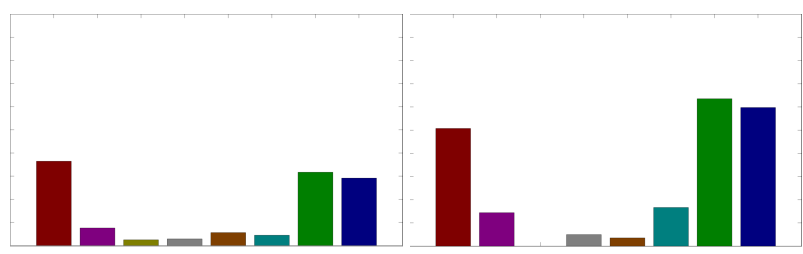

(c) On feature set $\boldsymbol{f}_{5}$ (Left), $\boldsymbol{f}_{6}$ (Right)

buiding

Figure 3: Accuracy of each class on each feature set, with each class is normalized to 100 .

Table 5: Average accuracy of RDF classifier on the feature sets. Feature sets $-\boldsymbol{f}_{i}$ mean the rest of all 6 feature sets except $\boldsymbol{f}_{i}$ is used, $i=1, \cdots, 6$.

\begin{tabular}{l|llllll}
\hline feature sets & $-\boldsymbol{f}_{1}$ & $-\boldsymbol{f}_{2}$ & $-\boldsymbol{f}_{3}$ & $-\boldsymbol{f}_{4}$ & $-\boldsymbol{f}_{5}$ & $-\boldsymbol{f}_{6}$ \\
\hline accuracy & $58.1 \%$ & $57.2 \%$ & $58.8 \%$ & $58.1 \%$ & $58.3 \%$ & $53.0 \%$ \\
\hline
\end{tabular}

From Fig. 3. we observe that each feature set performs reasonable results on building, window, and vegetation classes. Color features $\boldsymbol{f}_{2}$ perform better than other features on vegetation class because most vegetation parts are homogeneous regions. For other classes, each feature set performs not good. Relatively, Peucker features $\boldsymbol{f}_{4}$ perform better than other feature sets on minor classes. SIFT features $\boldsymbol{f}_{6}$ perform better than other features on average.

We also make the experiments using leave-one-out method. The overall classification accuracy is listed in Table 5 Feature sets $-\boldsymbol{f}_{i}$ mean the rest of all 6 feature sets except $\boldsymbol{f}_{i}$ is used, $i=$ $1, \cdots, 6$. The number of decision trees is chosen as $T=250$.

In the following, we make use of all the feature sets $\boldsymbol{f}_{1}, \boldsymbol{f}_{2}, \boldsymbol{f}_{3}$, $\boldsymbol{f}_{4}, \boldsymbol{f}_{5}$, and $\boldsymbol{f}_{6}$. We run experiments 5 times, and obtain overall averaging classification accuracy $58.8 \%$. The number of decision trees is also chosen as $T=250$. Fig. 4 Left shows the classification results over all 8 classes. The classification accuracy with respect to numbers of decision trees $T$ for training are shown in Fig. 4 Right. While increasing the number of decision trees, the classification accuracy also increases. After 250 iteration, the accuracy converges. So we choose $T=250$ for performing experiments above.

Fig. 5 and Fig. 6 present some result images of RDF method. The black regions in all the result images and ground truth images correspond to background. The qualitative inspection of the results

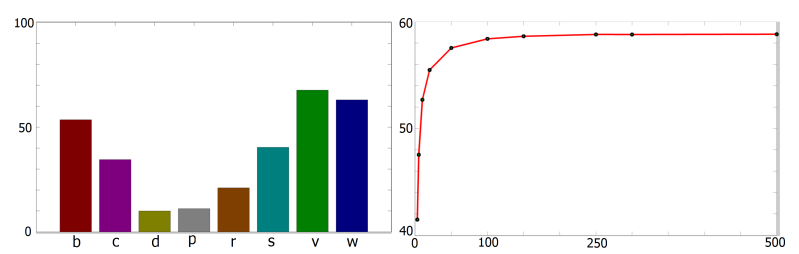

Figure 4: The classification accuracy of each class of the RDF classifier with mean shift and the accuracy with respect to the numbers of the decision trees. Left: the classification accuracy of each class on all feature sets. Right: the classification accuracy with respect to the numbers of the decision trees for training.

Table 6: Accuracy of RDF classifier with the mean shift segmentation on the eTRIMS 8-class dataset. The confusion matrix shows the classification accuracy for each class (rows) and is row-normalized to sum to $100 \%$. Row labels indicate the true class (Tr), and column labels the predicted class (Pr).

\begin{tabular}{|ll|llllllll|}
\hline $\mathrm{Pr}$ & $\mathrm{Tr}$ & $\mathrm{b}$ & $\mathrm{c}$ & $\mathrm{d}$ & $\mathrm{p}$ & $\mathrm{r}$ & $\mathrm{s}$ & $\mathrm{v}$ & $\mathrm{w}$ \\
\hline $\mathrm{b}$ & & $\mathbf{6 0}$ & 8 & 2 & 2 & 2 & 1 & 9 & 16 \\
$\mathrm{c}$ & 22 & $\mathbf{4 0}$ & 1 & 3 & 1 & 2 & 29 & 2 \\
$\mathrm{~d}$ & 46 & 0 & $\mathbf{1 5}$ & 0 & 0 & 0 & 8 & 31 \\
$\mathrm{p}$ & 40 & 16 & 0 & $\mathbf{1 2}$ & 4 & 4 & 16 & 8 \\
$\mathrm{r}$ & 40 & 20 & 0 & 14 & $\mathbf{2 3}$ & 3 & 0 & 0 \\
$\mathrm{~s}$ & 29 & 2 & 0 & 5 & 2 & $\mathbf{4 8}$ & 7 & 7 \\
$\mathrm{v}$ & 11 & 5 & 1 & 1 & 1 & 0 & $\mathbf{7 6}$ & 5 \\
$\mathrm{w}$ & 24 & 1 & 2 & 0 & 0 & 1 & 4 & $\mathbf{6 8}$ \\
\hline
\end{tabular}

in Fig. 5 shows that RDF classifier yields good results. In Fig.6 there exists some misclassification for each class. For example, the incorrect results at windows are often due to the reflectance of vegetation and sky in the window panes. Most sky regions are classified correctly. However, sky region is assigned label car in one image (last row in Fig. 6). This can be resolved simply by introducing some kind of spatial prior (Gould et al. 2008), such as sky is above the building, road and pavement are below the building, car is above the road, and window is surrounded by building.

A full confusion matrix summarizing RDF classification results over all 8 classes is given in Table 6 showing the performance of this method.

\subsection{Evaluation with watershed segmentation and RDF clas- sifier}

To test whether the classification result mainly benefits from the mean shift segmentation method, and not from the feature sets we use, we also employ another unsupervised segmentation method, namely the watershed algorithm by Vincent and Soille (1991), to segment the facade images.

The overall classification accuracy is $55.4 \%$, with the RDF classifier on all the feature sets and the number of the decision trees chosen as $T=250$. The confusion matrix is given in Table 7

In comparison with Table 6 the accuracy for each class remains similar, which shows that the type of finding image regions from the image segmentation algorithms is not critical and the low classification performance results from the lack of either good features or contextual information. 

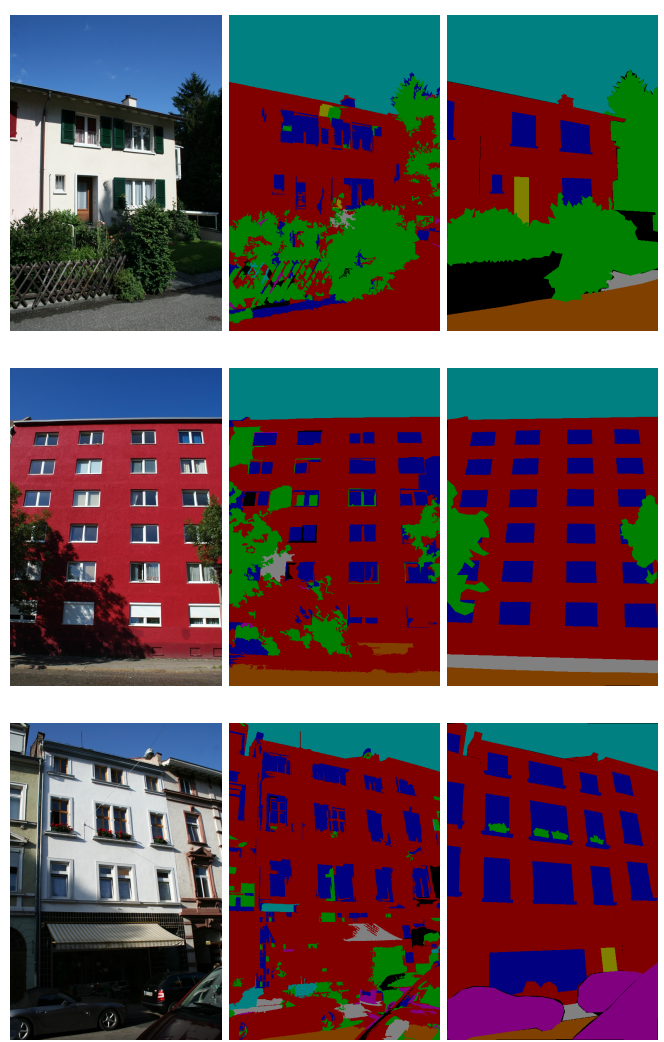

Figure 5: Qualitative classification results of a RDF classifier with the mean shift on the testing images from the eTRIMS dataset. (Left: test image, middle: result, right: ground truth.)
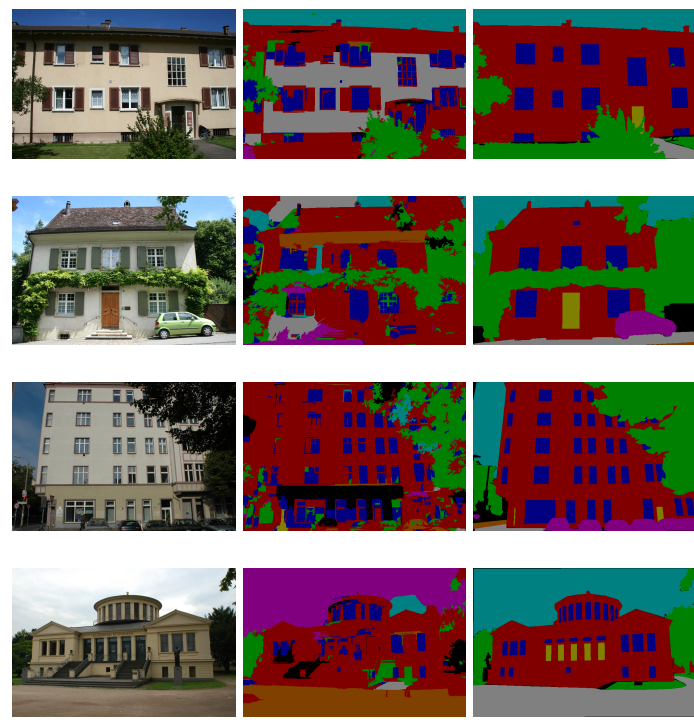

building

Figure 6: Some more classification results of a RDF classifier with the mean shift on the testing images from the eTRIMS dataset. (Left: test image, middle: result, right: ground truth.)
Table 7: Pixelwise accuracy of the image classificationusing the RDF classifier and the watershed segmentation on the eTRIMS 8 -class dataset. The confusion matrix shows the classification accuracy for each class (rows) and is row-normalized to sum to $100 \%$. Row labels indicate the true class $(\mathrm{Tr})$, and column labels the predicted class $(\mathrm{Pr})$.

\begin{tabular}{|ll|llllllll|}
\hline $\mathrm{Pr}$ & $\mathrm{Tr}$ & $\mathrm{b}$ & $\mathrm{c}$ & $\mathrm{d}$ & $\mathrm{p}$ & $\mathrm{r}$ & $\mathrm{s}$ & $\mathrm{v}$ & $\mathrm{w}$ \\
\hline $\mathrm{b}$ & & $\mathbf{5 9}$ & 4 & 1 & 3 & 5 & 9 & 11 & 7 \\
$\mathrm{c}$ & 67 & $\mathbf{2 1}$ & 0 & 5 & 2 & 0 & 3 & 2 \\
$\mathrm{~d}$ & 19 & 0 & $\mathbf{1 2}$ & 0 & 0 & 0 & 62 & 7 \\
$\mathrm{p}$ & 57 & 3 & 0 & $\mathbf{9}$ & 30 & 0 & 0 & 1 \\
$\mathrm{r}$ & 14 & 1 & 0 & 58 & $\mathbf{2 3}$ & 1 & 3 & 1 \\
$\mathrm{~s}$ & 17 & 0 & 0 & 6 & 0 & $\mathbf{7 3}$ & 2 & 1 \\
$\mathrm{v}$ & 13 & 4 & 1 & 2 & 1 & 13 & $\mathbf{6 1}$ & 4 \\
$\mathrm{w}$ & 29 & 1 & 1 & 1 & 0 & 6 & 3 & $\mathbf{5 7}$ \\
\hline
\end{tabular}

\subsection{Discussion}

With respect to the three most important classes building, window, and vegetation, we are satisfied with our classification results. But our multi-class approach does not perform very well for most of the other classes. Our classification scheme is faced with a dramatic inequality between the sizes of the classes. Almost $60 \%$ of the data is covered by only 2 classes, and the rest is spread over the rest classes. And for the classes like car and door, Gestalt features (Bileschi and Wolf. 2007) may play major role in a good classification performance. We also believe symmetry and repetition features are vital for classifying window class.

In this paper, features are extracted at local scale. Classification results are achieved from bottom up on these local features by classifiers. This factor leads to noisy boundaries in the example images. To enforce consistency, a Markov or conditional random field (Shotton et al. 2006) is often introduced for refinement, which would likely improve the performance.

\section{CONCLUSIONS}

We evaluate the performance of seven feature sets with respect to region-based classification of facade images. The feature sets include basic features, color features, histogram features, Peucker features, texture features, and SIFT features. We use randomized decision forest (RDF) to perform the classification scheme. In our experiments on the eTRIMS dataset (Korč and Förstner. 2009), we have shown that RDF produces some reasonable classification results.

The results show that these features and a local classifier are not sufficient. In order to recover more precise boundaries, the work presented in this paper has been fused into conditional random field framework (Yang and Förstner. 2011) by including neighboring region information in the pairwise potential of the model, which allows us to reduce misclassification that occurs near the edges of objects. As future work, we are interested in evaluating more features, such as Gestalt features (Bileschi and Wolf. 2007) and other descriptor features (van de Sande et al. 2010), for building facade images.

\section{References}

Barnard, K., Duygulu, P., Freitas, N. D., Forsyth, D., Blei, D. and Jordan, M., 2003. Matching words and pictures. Journal of Machine Learning Research 3, pp. 1107-1135. 
Bileschi, S. and Wolf, L., 2007. Image representations beyond histograms of gradients: The role of gestalt descriptors. In: IEEE Conference on Computer Vision and Pattern Recognition, pp. $1-8$

Bosch, A., Zisserman, A. and Muñoz, X., 2007. Image classification using random forests and ferns. In: IEEE International Conference on Computer Vision, pp. 1-8.

Breiman, L., 2001. Random forests. Machine Learning 45(1), pp. 5-32.

Comaniciu, D. and Meer, P., 2002. Mean shift: A robust approach toward feature space analysis. IEEE Transactions on Pattern Analysis and Machine Intelligence 24(5), pp. 603-619.

Dalal, N. and Triggs, B., 2005. Histograms of oriented gradients for human detection. In: IEEE Conference on Computer Vision and Pattern Recognition, pp. 886-893.

Drauschke, M. and Förstner, W., 2008. Selecting appropriate features for detecting buildings and building parts. In: Proceeding of the 21 st Congress of the International Society for Photogrammetry and Remote Sensing (ISPRS), pp. 447-452.

Drauschke, M. and Mayer, H., 2010. Evaluation of texture energies for classification of facade images. In: ISPRS Technical Commission III Symposium on Photogrammetry Computer Vision and Image Analysis, pp. 257-262.

Dumont, M., Marée, R., Wehenkel, L. and Geurts, P., 2009. Fast multi-class image annotation with random subwindows and multiple output randomized trees. In: International Conference on Computer Vision Theory and Applications (VISAPP), Vol. 2, pp. 196-203.

Felzenszwalb, P. F. and Huttenlocher, D. P., 2004. Efficient graph-based image segmentation. International Journal of Computer Vision 59(2), pp. 167-181.

Fröhlich, B., Rodner, E. and Denzler, J., 2010. A fast approach for pixelwise labeling of facade images. In: International Conference on Pattern Recognition, pp. 3029-3032.

Geurts, P., Ernst, D. and Wehenkel, L., 2006. Extremely randomized trees. Machine Learning 63(1), pp. 3-42.

Gould, S., Rodgers, J., Cohen, D., Elidan, G. and Koller, D., 2008. Multi-class segmentation with relative location prior. International Journal of Computer Vision 80(3), pp. 300-316.

Kluckner, S. and Bischof, H., 2010. Image-based building classification and $3 \mathrm{~d}$ modeling with super-pixels. In: ISPRS Technical Commission III Symposium on Photogrammetry Computer Vision and Image Analysis, pp. 233-238.

Korč, F. and Förstner, W., 2008. Interpreting terrestrial images of urban scenes using discriminative random fields. In: Proceeding of the 21st Congress of the International Society for Photogrammetry and Remote Sensing (ISPRS), pp. 291-296.

Korč, F. and Förstner, W., 2009. eTRIMS image database for interpreting images of man-made scenes. Technical Report TRIGG-P-2009-01.

Kumar, S. and Hebert, M., 2003. Discriminative random fields: a discriminative framework for contextual interaction in classification. In: IEEE International Conference on Computer Vision, pp. 1150-1157.

Lakatos, I., 1976. Proofs and Refutations. Cambridge: Cambridge University Press.
Lazaridis, G. and Petrou, M., 2006. Image registration using the Walsh transform. IEEE Transactions on Image Processing 15(8), pp. 2343-2357.

Lepetit, V., Lagger, P. and Fua, P., 2005. Randomized trees for real-time keypoint recognition. In: IEEE Conference on Computer Vision and Pattern Recognition, pp. 775-781.

Lowe, D., 2004. Distinctive image features from scale-invariant keypoints. International Journal of Computer Vision 60(2), pp. 91-110.

Maree, R., Geurts, P., Piater, J. and Wehenkel, L., 2005. Random subwindows for robust image classification. In: IEEE Conference on Computer Vision and Pattern Recognition, pp. 34-40.

Nister, D. and Stewenius, H., 2006. Scalable recognition with a vocabulary tree. In: IEEE Conference on Computer Vision and Pattern Recognition, pp. 2161-2168.

Petrou, M. and Bosdogianni, P., 1999. Image Processing: The Fundamentals. Wiley.

Rottensteiner, F., Trinder, J., Clode, S. and Kubik, K., 2007. Building detection by fusion of airborne laser scanner data and multi-spectral images: Performance evaluation and sensitivity analysis. ISPRS Journal of Photogrammetry and Remote Sensing 62(2), pp. 135-149.

Schnitzspan, P., Fritz, M. and Schiele, B., 2008. Hierarchical support vector random fields: joint training to combine local and global features. In: European Conference on Computer Vision, Part II, pp. 527-540.

Shotton, J., Johnson, M. and Cipolla, R., 2008. Semantic texton forests for image categorization and segmentation. In: IEEE Conference on Computer Vision and Pattern Recognition, pp. 1-8.

Shotton, J., Winnand, J., Rother, C. and Criminisi, A., 2006. Textonboost: joint appearance, shape and context modeling for multi-class object recognition and segmentation. In: European Conference on Computer Vision, Part I, pp. 1-15.

Sivic, J., Russell, B. C., Efros, A. A., Zisserman, A. and Freeman, W. T., 2005. Discovering objects and their locations in images. In: IEEE International Conference on Computer Vision, pp. 18.

Teboul, O., Simon, L., Koutsourakis, P. and Paragios, N., 2010. Segmentation of building facades using procedural shape priors. In: IEEE Conference on Computer Vision and Pattern Recognition, pp. 3105-3112.

van de Sande, K. E. A., Gevers, T. and Snoek, C. G. M., 2010. Evaluating color descriptors for object and scene recognition. IEEE Transactions on Pattern Analysis and Machine Intelligence 32(9), pp. 1582-1596.

Vedaldi, A. and Fulkerson, B., 2008. Vlfeat: An open and portable library of computer vision algorithms. http://www.vlfeat.org/.

Verbeek, J. and Triggs, B., 2007. Region classification with markov field aspect models. In: IEEE Conference on Computer Vision and Pattern Recognition, pp. 1-8.

Vincent, L. and Soille, P., 1991. Watersheds in digital spaces: An efficient algorithm based on immersion simulations. IEEE Transactions on Pattern Analysis and Machine Intelligence 13(6), pp. 583-598.

Yang, M. Y. and Förstner, W., 2011. Regionwise classification of building facade images. In: Photogrammetric Image Analysis (PIA2011), LNCS 6952, Springer, pp. 209 - 220. 